\title{
is Research Square \\ FLASH irradiator at the Radiological Research Accelerator Facility.
}

Guy Garty ( $\sim$ gyg2101@cumc.columbia.edu )

Columbia University Medical Center

\section{Razib Obaid}

Columbia University Medical Center

Naresh Deoli

Columbia University Medical Center

Ekaterina Royba

Columbia University Medical Center

Andrew D. harken

Columbia University Medical Center

David J. Brenner

Columbia University Medical Center

\section{Research Article}

Keywords:

Posted Date: January 21st, 2022

DOI: https://doi.org/10.21203/rs.3.rs-1281287/v1

License: (c) (i) This work is licensed under a Creative Commons Attribution 4.0 International License.

Read Full License

Version of Record: A version of this preprint was published at Scientific Reports on December 22nd, 2022. See the published version at https://doi.org/10.1038/s41598-022-19211-7. 


\section{Abstract}

The Radiological Research Accelerator Facility has modified a decommissioned Varian Clinac to deliver FLASH dose rates:

Operating in $9 \mathrm{MeV}$ electron mode (FLASH mode), samples can be irradiated inside the Clinac head at average dose rates of up to $600 \mathrm{~Gy} / \mathrm{sec}$ (3 Gy per $0.5 \mu \mathrm{sec}$ pulse, 180 pulses per sec). In this mode multiple pulses are required for most irradiations. By modulating pulse repetition rate, dose rates below 1 $\mathrm{Gy} / \mathrm{min}$ can be achieved, allowing comparison of FLASH and conventional irradiations with the same beam.

Operating in $6 \mathrm{MV}$ photon mode, with the conversion target removed (superFLASH mode), samples are irradiated at instantaneous dose rates of up to $1 \mathrm{GGy} / \mathrm{sec}$ (0.2-150 Gy per pulse, 360 pulses per sec) and most irradiations can be performed with a single very high dose rate pulse.

In both modes we have seen the expected inverse relation between dose rate and irradiated area, with the highest dose rates obtained for beams with a FWHM of about $2 \mathrm{~cm}$ and $\pm 10 \%$ uniformity over $1 \mathrm{~cm}$ diameter.

As an example of operation of the FLASH irradiator, we present dose rate dependence of dicentric chromosome yields.

\section{Introduction}

Dose rate effects have come under intense scrutiny over the last few years. In the context of radiation oncology, FLASH radiotherapy [1] makes use of very high dose rate ( $>50 \mathrm{~Gy} / \mathrm{sec}$ ) irradiations to reduce radiation effects in normal tissue, while providing the same level of tumor killing. Though the underlying mechanisms are not yet clear [2], the resulting enhanced therapeutic ratio has been observed in both animal [3] and human [4] studies.

In the context of biodosimetry, Improvised Nuclear Device (IND) exposures result in a broad range of dose rates [5]. As the main photon dose from a nuclear detonation is delivered in the form of prompt gamma rays, formed in the fraction of a microsecond before the fissile material is dispersed in the explosion [6], it is critical to test assay performance using a realistic exposure time scale, rather than the common " 1 Gy/min".

In general, low dose rates result in increased repair of sub-lethal damage, with SSB and DSB repair occurring in a few minutes or hours respectively [7], resulting in reduced DNA damage yields per unit dose. At high dose rates ( $\mathrm{Gy} / \mathrm{sec})$, some direct experiments [8], and measurements of fast DSB repair times [9] suggest that there will be increased effects from a dose delivered in $\sim 1 \mathrm{~s}$ compared with the same dose delivered in $\sim 1 \mathrm{~min}$. Conversely, the FLASH effect would seem to indicate that at very high doses and dose rates, where oxygen depletion in the cellular environment plays a major role in radiation 
response, there may be a decline in DNA damage, formed in an oxygen-dependent processes. It thus becomes crucial to develop irradiation platforms that could deliver an LD50 dose, in $\mu s e c$ (modeling the blast) in addition to days (modeling fallout).

High dose rate is also extremely useful when modeling partial body exposures in mice where the irradiation should be significantly faster than the circulation time of blood in the mouse ( $15 \mathrm{sec}[10])$. Otherwise, instead of irradiating a fraction $(f)$ of the blood to a given dose $(D)$, all the circulating blood is irradiated to dose $D / f$ - a completely different scenario.

Having recently developed irradiation platforms to study low dose rate effects [11, 12], we describe here a dedicated FLASH irradiator, that we have implemented at the Radiological Research Accelerator Facility (RARAF). The irradiator is based on a decommissioned medical linear accelerator (linac), similar to the platforms developed at Stanford [13] and Dartmouth [14]. As opposed to the prior systems described in the literature, we are not using a clinically-active machine. We were thus able to make modifications incompatible with future patient use.

As described below, our FLASH irradiator allows irradiation of mice, cells, and blood vials at dose rates ranging from conventional (Gy/min) to $>100$ Gy per $0.1 \mu$ sec linac pulse.

As an example of the studies now open to us, we demonstrate reduced dicentric yields at ultrahigh dose rates. A separate manuscript (Padilla, in process) describes FLASH treatment of Glioma in mice.

\section{Methods}

\section{Platform development}

The RARAF FLASH irradiator is based on a retired Clinac 2100C (Varian Medical Systems, Palo Alto, CA), recently installed in the accelerator hall at RARAF (Fig. 1). Due to the tight space, the bed was discarded, and the gantry rotation was disabled with the beam permanently pointing vertically up.

For most FLASH irradiations, the Clinac is operated in service mode, using the $9 \mathrm{MeV}$ electron setting (FLASH mode), providing the best penetration into tissue below the $11 \mathrm{MeV}$ photoneutron threshold of ${ }^{56} \mathrm{Fe}$ [15]. Higher dose rates, with a degraded dose depth profile are achievable when operating in $6 \mathrm{MV}$ photon mode, with the target retracted (SuperFLASH mode). In this mode, the electron current is increased by several orders of magnitude, nominally to overcome the low photon yield at $6 \mathrm{MeV}$, allowing very high electron dose rates.

To minimize beam divergence, the foil assembly was removed from the carousel. The target piston was also decoupled from the Clinac control hardware and was set to be permanently in the "out" position. In this way the only material in the beam is the beam exit window and the integrated ionization chamber assembly. 
We have modified the timer interface card (Fig. 2), replacing the Varian generated GDLY CNT signal with our own signal. Under normal Clinac operation, this signal controls the delay between the electron gun firing and the klystron. When GDLY CNT is 0 , the two are synchronized and radiation is delivered; when it is $+12 \mathrm{~V}$ the electron beam is generated out of phase with the klystron and no radiation is delivered.

To generate the control pulse train (Fig. 3) we utilized a USB-CTR08 card (Measurement Computing, Norton, MA). The card accepts as input the KLY I signal from the Clinac controller and turns the output on $(+5 \mathrm{~V})$ after a predetermined number of $\mathrm{KLY}$ I pulses for a predetermined number of pulses and then either stops or repeats. The output signal is used to actuate a solid-state relay (VOR1121A6; Mouser Electronics, Mansfield, TX) which grounds TP21 on the timer Interface card (beam on) or allows it to be pulled up to $+12 \mathrm{~V}$ (beam off).

\section{Dosimetry}

In $9 \mathrm{MeV}$ electron mode, beam intensity could be monitored using the built-in ion chamber. Specifically, test Point TP1 on the ion chamber control card was hooked up to a multichannel analyzer, and the average pulse height and number of pulses recorded for each irradiation. In superFLASH mode, the ion chamber is severely saturated and cannot be used (Fig. 4). An alternative beam monitor is under development.

NIST traceable dosimetry was performed using an Advanced Markus ion chamber (AMIC) and UNIDOS E electrometer (PTW, Freiburg, Germany). The ion chamber was calibrated to absorbed dose in water, using $a^{60}$ Co source at the MD Anderson Accredited Dosimetry Calibration Laboratory (ADCL). Measured dose was corrected for temperature, pressure, and radiation quality (Table 1).

However, the AMIC only provides reliable dosimetry for dose rates of up to about $1.5 \mathrm{kGy} / \mathrm{sec}$ and for radiation fields $>1 \mathrm{~cm}$ in diameter. For higher dose rates and smaller fields, we used EBT3 Radiochromic film (Ashland Specialty Chemicalsf, Wayne NJ) at doses below 20 Gy and OC-1 OrthoChromic film (Orthochrome Inc., Hillsborough, $\mathrm{NJ}$ ) at doses above $5 \mathrm{~Gy}$. Each batch of film was calibrated by irradiating film and the AMIC at a dose rate of approximately $1 \mathrm{~Gy} / \mathrm{sec}$, using a large field size (Fig. S1).

Film was scanned using an Epson V700 scanner in transmission mode (EBT-3) or reflection mode (OC-1). The scanner was operated several times to warm up the lamp and then films (including an unirradiated control film) were placed on the scanner bed (EBT-3 under a sheet of glass) and scanned at $300 \mathrm{DPI}$ and a bit depth of 48 bit. Only the red channel was used for dose reconstruction. Optical density was taken as negative $\log 10$ of the ratio of red color value in the irradiated and unirradiated films. Dose vs. optical density was calibrated using a first order rational function $D=\frac{a-b \times O D}{O D-c}$. A matlab script was then used to convert scanned film images to dose maps and to generate dose histograms in selected regions of the image.

\section{Safety}


Our accelerator hall was originally built of interlocking 27 " thick reinforced concrete shield blocks. As this is not strictly sufficient shielding in conventional clinical operations [16], we have mapped the leakage radiation and lock off high radiation rooms (specifically the room directly above the Clinac head) during operation. Lower radiation areas are cordoned off as "no linger" zones. Similar to clinical installations, the DOOR interlock is used to ensure that the accelerator hall is vacated and locked during use. A GeigerMuller based area monitor (Luldlum Measurements Inc, Sweetwater, TX) is located adjacent to the Clinac with a lighted sign visible from outside the accelerator hall. Additional monitors are placed in the rooms adjacent to the vault, including at the console.

As we are only irradiating using electrons and have seen excessive stray radiation outside the accelerator hall only when generating photon beams, we have disabled the target piston, such that the target is always in the out position.

\section{Sample positioning}

To allow for reproducible sample positioning both inside and outside the Clinac head, we have constructed a scaffold, using $25 \mathrm{~mm}$ extruded aluminum optical construction rails (Thorlabs, Newton, $\mathrm{NJ}$ ). A graduated optical dovetail rail (Thorlabs) was bolted on the construction rail with the sample holder mounted on a dovetail rail carrier (Fig. 5b). Test tubes were placed in a 1.5" bored acrylic cube (McMaster-Carr, Chicago, IL; Fig. 5a/c). In addition, a custom holder was designed and 3D printed (Protolabs Inc, Maple Plains, MN) using PA12 Nylon (Fig. 5d). This holder allowed irradiation of larger objects, Petri dishes or a mouse anesthesia jig (Precision X ray Irradiation Inc, North Branford, CT; Fig. 5 e). Adjustable $1 / 4 "$ lead screens were added for beam collimation.

To facilitate sample alignment to the beam we have mounted a $5 \mathrm{~mW}$ red cross projection laser (Laserglow Technologies, North York, ON) to the top of the scaffold. The laser was aligned to the beam using in situ irradiated film.

\section{Dicentric analysis}

This study was approved by Columbia University's Institutional Review Board (IRB) protocol IRBAAAR0643. Peripheral blood was obtained after informed consent from two healthy volunteers (male, 32 yr. and female, 54 y.o.) with no recent history of exposure to ionizing radiation or clastogenic agents. Freshly-drawn blood was heparinized (vacutainers with sodium heparin anticoagulant (Becton Dickinson, $\mathrm{NJ})$ ), aliquoted into Matrix Storage Tubes (Fisher), and exposed to $3 \mathrm{~Gy}$ or $8 \mathrm{~Gy} 9-\mathrm{MeV}$ electrons at a wide range of the dose-rates $\left(3 \times 10^{5} \mathrm{~Gy} / \mathrm{sec}(3 \mathrm{~Gy}\right.$ only), $600 \mathrm{~Gy} / \mathrm{sec}, 50 \mathrm{~Gy} / \mathrm{sec}, 5 \mathrm{~Gy} / \mathrm{sec}$, and $1 \mathrm{~Gy} / \mathrm{min})$. The control samples were sham-irradiated and received 0 Gy dose.

After irradiation, the blood $(0.5 \mathrm{~mL})$ was stimulated with a mitogen (phytohemagglutinin, PB-MAX karyotyping medium) and incubated at $37^{\circ} \mathrm{C}, 5 \% \mathrm{CO}^{2}$ for $48 \mathrm{hrs} .3 \mathrm{hrs}$ before fixation, cells were arrested in metaphase with $0.1 \mathrm{mg} / \mathrm{ml}$ colcemid (Gibco). Following the treatment, cells were swollen for $20 \mathrm{~min}$ in hypotonic solution (0.075 M KCl) and fixed with methanol:acetic acid (3:1). $20 \mu \mathrm{l}$ of fixed cells were dropped onto glass slides, dried at an ambient temperature of $25^{\circ} \mathrm{C}$ and relative humidity of $55 \%$. Dried 
slides were then fixed in $4 \%$ formaldehyde and dehydrated in ethanol (70\%-85\%-100\% for 2 min. each). Prepared chromosome spreads were stained with a PNA probe hybridization cocktail (FITC-labelled centromere and Cy3-labelled telomere probes (PNABio) in buffer), covered by a cover glass, denatured for $3 \mathrm{~min}$. at $80^{\circ} \mathrm{C}$, and left at a room temperature in the dark for $2 \mathrm{hrs}$. After that, the slides were washed twice in $70 \%$ formamide, then twice in TBST (with $0.05 \%$ Tween $^{\mathrm{TM}}-20$ ), and counterstained with Vectashield® Antifade Mounting Medium with DAPI (4',6-diamidino-2-phenylindole).

Images were acquired on a Metafer 4 Scanning System (MetaSystems) equipped with a Carl Zeiss Axioplan Imager Z1, a CoolCube 1 Digital High-Resolution CCD Camera, and a Zeiss Plan-Apochromat $63 x$ oil immersion objective. Metaphases were located using an automated classifier comprised of two acquisition modules, MSearch and AutoCapt. Captured images were analyzed using Isis software (MetaSystems). Metaphase cells were selected at low magnification (x10) and examined for quality under higher magnification (x63) to exclude cells with overlapped chromosomes or not clearly distinguishable chromatids. Only complete metaphases (46 centromere spots) were used for analysis. The samples were blinded to the scorer and decoded afterward. For scoring, 50 cells per donor were analyzed (QuickScan method [17]) using the morphological criteria specified by IAEA cytogenetic dosimetry manual [18]. The dicentric yields were subsequently adjusted by conversion of multicentric aberrations into the dicentric equivalent (tricentric chromosome is equal to two dicentrics, tetracentric chromosome is equal to three dicentrics, etc.). The yields of dicentrics and their distribution among cells have been used to calculate the dispersion index $\left(\sigma^{2} / y\right)$ and the normalized unit of this index $(u)$ using the equation recommended by IAEA [18].

For dose-response curves, the yields of dicentrics $(Y)$ was used to calculate the coefficients of the linearquadratic mathematical function $\left(Y=\alpha D+\beta D^{2}\right.$, where $D$ is a dose and $a$ and $\beta$ are the linear and quadratic coefficients, respectively) in CABAS v.2 software [19]. The uncertainties for $\alpha / \beta$ quotients were estimated by propagating the relative errors in quadrature: $\sigma_{\alpha / \beta}=\frac{\alpha}{\beta} \sqrt{\left(\frac{\sigma_{\alpha}}{\alpha}\right)^{2}+\left(\frac{\sigma_{\beta}}{\beta}\right)^{2}}$, where $\sigma$ represents the standard error.

\section{Results}

\section{Reproducibility}

Figure 6 shows the average pulse height measured over a set of 16 experiments conducted in the 2 nd half of 2021. In each experiment, 4-10 samples were irradiated using 2 pulses and 4-10 using 3 pulses. The graph shows fluctuations of $1-2 \%$ (relative standard deviation) in pulse height within a set of irradiations and a slow drift over several months.

In superFLASH mode, the built-in IC always reads the same, saturated, pulse height, so reproducibility can only be tested using film. Based on a smaller set of film measurements, pulse height variations were roughly $\pm 15 \%$ within a single run. 


\section{Beam characterization}

Pulse timing

Figure 4 (above) shows an oscilloscope trace of the built-in IC signal for 1 pulse in FLASH and superFLASH modes. The pulse width is dominated by the ion chamber shaping electronics and cannot be used to estimate the actual pulse duration. Using a nanosecond timing scintillator (EJ-301; ELJEN Technology, Sweetwater, TX) and photomultiplier tube (RCA 8575), we were able to measure pulse width as $0.13 \mu \mathrm{sec}$ FWHM (Fig. 7) in FLASH mode. In superFLASH mode we have seen saturation of the PMT resulting in a significantly broadened pulse.

Percent Depth dose

PDD was measured using radiochromic film sandwiched between sheets of water equivalent polystyrene (RW3, LAP laser, Boynton Beach, FL). The film was placed in the plane of the beam, below the $\mathrm{X}$ jaws and irradiated with a single pulse of 6 or $9 \mathrm{MeV}$ electrons (Fig. 8). At this position, the beam diameter is about $2 \mathrm{~cm}$. $R_{50}$ is $40 \mathrm{~mm}$ for $9 \mathrm{MeV}$ and $20 \mathrm{~mm}$ for $6 \mathrm{MeV}$. It is also obvious from Fig. 8a,b that the $6 \mathrm{MeV}$ beam scatters significantly more than the $9 \mathrm{MeV}$ beam.

Dose rate vs spot size

Figure 9 and Supplementary Figure 2 show 6 and $9 \mathrm{MeV}$ electron beam brightness at various positions in the system, with the isocenter at $1000 \mathrm{~mm}$.

\section{Dicentric analysis}

The numbers and distribution of dicentrics among cells are given in Fig. 10 and Supplementary Table 1. The resulting dose-response curves are shown in Supplementary Figure 3. The $\alpha$ and $\beta$ coefficients, and the $a / \beta$ quotients for each dose rate are given in Table 2 .

\section{Discussion}

\section{FLASH platform operation}

To date, the FLASH platform at RARAF has been operational for over a year and a half. After initial optimization of the beam settings, we have been able to operate with minimal adjustments. As seen from Fig. 6, reproducibility of the beam intensity is quite good. The slow decline is suspected to be related to aging of the high voltage diodes in the DeQing circuit driving the klystron. We have seen increased beam brightness when these diodes were replaced in spring of 2021. It should be noted that we have seen good correlation between the ion chamber signal and dose measured using film or the AMIC.

\section{Optimization of beam delivery}


For each experiment, we optimized the sample position, pulse structure, and delivered number of pulses, while monitoring both the AMIC and the pulse height spectrum from the built-in ion chamber. In general, we have seen a good correlation between the delivered dose, at a given position, and the integral of the pulse height spectrum.

When operating in the $9 \mathrm{MeV}$ electron mode, we have seen that the optimal beam brightness is obtained when allowing the electron gun to run for $20 \mathrm{sec}$ (3600 pulses) prior to generating the beam. Anecdotally, when delivering short $(<1 \mathrm{sec})$ bursts, reproducibility in beam intensity seems to be better if there is a 2-3minute gap between irradiations.

\section{Conventional dose rate}

For irradiation at conventional dose rates (1-2 Gy/min), samples were placed at $1.65 \mathrm{~m}$ above the head ( $65 \mathrm{~cm}$ beyond the isocenter). At this position, the nominal dose rate is about $0.01 \mathrm{~Gy} / \mathrm{pulse}$ and the field size is large, so that several test tubes, a flask or a multiwell plate could be placed alongside the AMIC. The electrometer was operated at medium scale and the pulse repetition rate was adjusted to 50-100 pulses off for every on pulse, such that the AMIC was reading the required dose rate. Repetition rate was adjusted periodically to compensate for drift in the delivered dose per pulse. Beam was stopped manually when the desired dose was achieved, typically with a precision better than $0.1 \mathrm{~Gy}$.

Moderately high dose rate

For dose rates of approximately $1 \mathrm{~Gy} / \mathrm{sec}$, human reflexes (and the refresh rate of the UNIDOSE E) are not fast enough to allow stopping the beam manually and there is sufficient drift in the beam intensity that delivering a known number of pulses does not necessarily result in the exact required dose.

To deliver a predetermined dose at $1 \mathrm{~Gy} / \mathrm{sec}$ we utilized the DOS1 interlock. Samples were irradiated at a pulse repetition rate of $180 \mathrm{~Hz}$ (the maximal repetition rate for $9 \mathrm{MeV}$ electrons), in the same geometry as the conventional dose rate samples. We first irradiated the AMIC alone to determine the correct Monitor Unit (MU) count for the prescribed dose (25-30 MU/Gy with small day-to-day variations). We then irradiated the sample and AMIC side by side with our software set to deliver an excess of pulses and the DOS1 interlock set to trip at the required MU count. This typically resulted in a few $\%$ accuracy in delivered dose.

High dose rate

Dose rates of 20-100 Gy/sec could be delivered with the sample placed inside the Clinac head (roughly in the position of the MLC or Y jaw). The field size in this case is too small for irradiating the sample and the AMIC simultaneously. Prior to each set of irradiations, the AMIC was used to find the position within the head and the required number of pulses for the prescribed dose rate and dose.

Typically, beam was delivered by waiting $20 \mathrm{sec}$ with the klystron and electron gun on but out of synchronization and then delivering the required number of pulses. This resulted in a maximal average 
pulse height from the built-in ion chamber, although pulse height was seen to start drifting after about a second of beam-on time.

Very high dose rates

With the sample at or below the $X$ jaw, average dose rates of $200-900 \mathrm{~Gy} / \mathrm{sec}(1-5 \mathrm{~Gy} / \mathrm{pulse})$ could be obtained using $9 \mathrm{MeV}$ electrons. In this case dosimetry cannot be done using the AMIC at all, as the beam size is comparable to the AMIC sensitive volume and instantaneous dose rate is much higher than the 1.5 $\mathrm{kGy} / \mathrm{sec}$ rating of the AMIC. We, therefore, determined dose rate by irradiating EBT3 film. As above, beam was delivered using a $20 \mathrm{sec}$ delay preceding the radiation pulses.

superFLASH mode

Even higher dose rates could be achieved when operating the Clinac in $6 \mathrm{MeV}$ photon mode, with the target removed. In this case the beam repetition rate is $360 \mathrm{~Hz}$ and the dose per pulse is also significantly higher (up to $150 \mathrm{~Gy} /$ pulse below the $X$ Jaw). Dosimetry was similarly performed using OC-1 film, which has better high dose performance, as compared to EBT3.

\section{Selection of dose rate}

The electron beam from the Clinac has a gaussian profile (Supplementary Figure 2) with the beam diameter increasing linearly with distance from the Clinac head. Figure 12 shows the inverse square relation between dose rate and beam diameter. When designing an experiment, our goal is to obtain the maximal dose rate while maintaining $< \pm 10 \%$ variation in beam intensity across the sample. This limits the maximal dose rate (in FLASH mode) depending on target size. Thus, a matrix tube ( $1 \mathrm{~cm}$ diameter) can be irradiated to a maximal dose of $3 \mathrm{~Gy} /$ pulse. Larger objects like $35 \mathrm{~mm}$ Petri dishes or T12.5 flasks are limited to doses of about $0.25 \mathrm{~Gy} /$ pulse and so on. In particular, mouse TBI is only available at dose rates of up to about $10 \mathrm{~Gy} / \mathrm{sec}$ using $9 \mathrm{MeV}$ electrons.

\section{Depth-Dose}

One potential problem with electron-based FLASH irradiations is the shallow penetration of electron beams, compared to photon beams. As seen in Fig. 8 and Table 1 above, the penetration of electron beams available to us are only a few $\mathrm{cm}$. This is particularly problematic with the SuperFLASH beam which only penetrates about $2 \mathrm{~cm}$ in tissue. For murine tumor irradiations, this is generally not a problem as the mouse can always be oriented such that the tumor is within the beam. Similarly, for irradiation of liquid samples (blood or cell cultures), proper selection of the aliquot size and vial shape can ensure that the entire sample is being irradiated to a uniform dose. When total-body-irradiating mice, however, the SuperFLASH mode may induce a large variation in dose in the mouse. For mouse TBI irradiations we are, therefore, testing alternative mouse holders where the mouse is splayed and slightly thinner than the conventional mouse holder (Fig. 5e). Initial tests have shown that at least with younger mice (7 week) the entrance and exit doses are within $10 \%$ of each other. 


\section{Dicentric Yields}

Overall, the detailed aberration analyses revealed a marked decrease in dicentric yields at higher dose rates ( $p$-values $<0.05$, estimated using T-test assuming equal (independent) variances). This is consistent with the so-called FLASH effect seen in many biological endpoints[1, 2].

Our data suggest that, at a conventional dose rate of $1 \mathrm{~Gy} / \mathrm{min}, 9 \mathrm{MeV}$ electrons induced more damage than ${ }^{137} \mathrm{Cs}$ gamma-rays (manually scored previously [20]). At $8 \mathrm{~Gy},{ }^{137} \mathrm{Cs}$ gamma-rays induced 2.6 dicentrics/cell while $9 \mathrm{MeV}$ electrons induced 8.1 dicentrics/cell. Moreover, at $8 \mathrm{~Gy}$, the aberrant chromosomes displayed tendencies to form very long multicentrics, with 4, 5, and 6 centromeres per chromosome (Notes to Supplementary Table 1). Dicentrics were distributed unevenly: at $8 \mathrm{~Gy} 9 \mathrm{MeV}$ electrons resulted in approximately 3 to 14 dicentrics/cell. This was higher than for $8 \mathrm{~Gy}{ }^{137} \mathrm{Cs}$ gammarays (1-9 dicentrics/cell). At higher dose rates (5 Gy/sec and $50 \mathrm{~Gy} / \mathrm{sec}$ ), the dicentric yields reduced, however, multicentrics were still predominant over dicentrics (Notes to Supplementary Table 1). When the dose rate increased up to $600 \mathrm{~Gy} / \mathrm{sec}$ (dose delivered in 3 pulses), the total numbers of dicentrics decreased even more, and aberrant chromosomes became shorter (mostly 2 centromeres per chromosome).

In general, the samples were in good agreement with the Poisson distribution, with only three samples where $u$ exceeded 1.96. Negative u-values, indicating underdispersion, were recorded for the samples exposed to $3 \mathrm{~Gy}(\mathrm{u}=-2.33)$ and $8 \mathrm{~Gy}(\mathrm{u}=-2.46)$ at a dose rate of $5 \mathrm{~Gy} / \mathrm{sec}$. The underdispersion could be due to a small number of donors and scored cells. The $u$-value was positive $(u=2.4)$ for $3 \mathrm{~Gy}$ sample exposed at a dose rate of $50 \mathrm{~Gy} / \mathrm{sec}$, implying overdispersion. Dicentric overdispersion was reported earlier for radiotherapy patients subjected to higher dose rate exposures, suggesting that the proportion of undamaged lymphocytes could increase with the dose rate (4). However, higher dose rates may not be the reason that caused overdispersion in our experiment because we did not observe the same effect for 8 Gy sample exposed at a same dose rate of $50 \mathrm{~Gy} / \mathrm{sec}$.

The relationships between dose and dicentric yield at different dose rates were analyzed using the function $Y=\alpha D+\beta D^{2}$ (Supplementary Figure 3). A representation of this function is that dicentrics require two lesions for their formation, which are induced by one or two tracks. The a and $\beta$ values shown in Table 3 indicate that a coefficients were similar (due to high errors), while the differences in the observed dicentric yields at higher dose rates were almost entirely due to the marked decrease in $\beta$ coefficients (and small errors). This supports the consideration, that only $\beta$ coefficient is modulated by the dose rate changes. As the values $\mathrm{a} / \beta$ are $0.4 \mathrm{~Gy}$ and $2.6 \mathrm{~Gy}, 1.8 \mathrm{~Gy}$, or $8.7 \mathrm{~Gy}$ for dicentrics produced at conventional $(1 \mathrm{~Gy} / \mathrm{min})$ vs. higher dose rates. This may indicate that sub-lethal damage is modulated to a much higher degree than lethal damage, at high dose rates and thus dicentrics produced by single tracks will predominate.

Experiments with a large cohort of volunteers is under way to investigate age and sex effects on the dose rate response of dicentric yields, using both high dose rates, using this platform, and very low dose rates, 
mimicking fallout [11].

\section{Conclusions}

We have developed a high dose rate irradiator based on a retired Varian Clinac 2100C. It has been in operation for almost two years performing both radiation oncology-related studies at dose rates of up to $100 \mathrm{~Gy} / \mathrm{sec}$ and biodosimetry-related studies at dose rates of up to $30 \mathrm{GGy} / \mathrm{sec}$.

In these investigations we have seen a marked reduction in dicentric yields in ex vivo irradiated blood at high dose rates.

\section{Declarations}

Declarations of interest: none

\section{Author contributions}

GG, DJB and RO designed and built the FLASH system. GG, DJB and ND designed and built the SuperFLASH system. GG, RO, ND and ADH performed the irradiations and beam diagnostics. All authors participated in writing the manuscript.

\section{Acknowledgements}

This work was partially supported by grant number U19 AI067773, and contract number HHSN272201600040C, from the National Institute of Allergy and Infectious Diseases (NIAID), National Institutes of Health $(\mathrm{NIH})$. Installation of the Clinac at RARAF was supported by the Radiation Oncology departments at Columbia University Irving Medical Center and Cornell medicine and by an unrestricted research gift from Barry Neustein. The authors thank Jim Sharkey, Ron Drake and James Viera for their assistance with Clinac setup and debugging.

\section{References}

1. Vozenin, M.C., J.H. Hendry, and C.L. Limoli, Biological Benefits of Ultra-high Dose Rate FLASH Radiotherapy: Sleeping Beauty Awoken. Clinical oncology (Royal College of Radiologists (Great Britain)), 2019. 31(7): p. 407-415.

2. Friedl, A.A., et al., Radiobiology of the FLASH effect. Med Phys, 2021.

3. Vozenin, M.C., et al., The Advantage of FLASH Radiotherapy Confirmed in Mini-pig and Cat-cancer Patients. Clin Cancer Res, 2019. 25(1): p. 35-42.

4. Bourhis, J., et al., Treatment of a first patient with FLASH-radiotherapy. Radiotherapy and Oncology, 2019. 139: p. 18-22. 
5. Garty, G., et al., Mice and the A-Bomb: Irradiation Systems for Realistic Exposure Scenarios. Radiation Research, 2017. 187(4): p. 475-485.

6. Glasstone, S. and P.J. Dolan, The Effects of Nuclear Weapons. Third edition. 1977; Department of Defense, Washington, D.C. (USA); Department of Energy, Washington, D.C. (USA). p. 660.

7. Sharma, P.M., et al., High throughput measurement of $y-H 2 A X$ DSB repair kinetics in a healthy human population PLOS One 2015. 10(3): p. e0121083.

8. Ling, C.C., I.J. Spiro, and R. Stickler, Dose-rate effect between 1 and $10 \mathrm{~Gy} / \mathrm{min}$ in mammalian cell culture. Br J Radiol, 1984. 57(680): p. 723-8.

9. Reynolds, P., et al., The dynamics of Ku70/80 and DNA-PKCs at DSBs induced by ionizing radiation is dependent on the complexity of damage. Nucleic Acids Res, 2012. 40(21): p. 10821-31.

10. Debbage, P.L., et al., Lectin intravital perfusion studies in tumor-bearing mice: micrometer-resolution, wide-area mapping of microvascular labeling, distinguishing efficiently and inefficiently perfused microregions in the tumor. J Histochem Cytochem, 1998. 46(5): p. 627-39.

11. Garty, G., et al., VADER: a variable dose-rate external 137Cs irradiator for internal emitter and low dose rate studies. Scientific Reports, 2020. 10(1): p. 19899.

12. Bertucci, A., et al., In vitro RABiT measurement of dose rate effects on radiation induction of micronuclei in human peripheral blood lymphocytes. Radiation and Environmental Biophysics, 2016: p. 1-7.

13. Schüler, E., et al., Experimental Platform for Ultra-high Dose Rate FLASH Irradiation of Small Animals Using a Clinical Linear Accelerator. International Journal of Radiation Oncology*Biology*Physics, 2017. 97(1): p. 195-203.

14. Rahman, M., et al., Electron FLASH Delivery at Treatment Room Isocenter for Efficient Reversible Conversion of a Clinical LINAC. International Journal of Radiation Oncology*Biology*Physics, 2021. 110(3): p. 872-882.

15. Tobin, R., J. McElhinney, and L. Cohen, Photoneutron Thresholds for Iron, Germanium, Rubidium, and Hafnium. Physical Review, 1958. 110(6): p. 1388-1391.

16. National Council on Radiation Protection \& Measurements, Structural Shielding Design and Evaluation for Megavoltage X-and Gamma-Ray Radiotherapy Facilities. 2005: Bethesda, MD.

17. Flegal, F.N., et al., Quickscan dicentric chromosome analysis for radiation biodosimetry. Health Phys, 2010. 98(2): p. 276-81.

18. International Atomic Energy Agency, Cytogenetic analysis for radiation dose assessment: a manual. 2001, Vienna: IAEA. 127.

19. Deperas, J., et al., CABAS: a freely available PC program for fitting calibration curves in chromosome aberration dosimetry. Radiat Prot Dosimetry, 2007. 124(2): p. 115-23.

20. Royba, E., et al., RABiT-II-DCA: A Fully-automated Dicentric Chromosome Assay in Multiwell Plates. Radiation research, 2019. 192(3): p. 311-323. 
21. Ainsbury, E.A. and D.C. Lloyd, Dose estimation software for radiation biodosimetry. Health physics, 2010. 98(2): p. 290-295.

\section{Tables}

Table 1: beam quality correction for the AMIC.

\begin{tabular}{|llll|}
\hline Mode & Energy & $R_{50}$ & \\
\hline superFLASH & $6 \mathrm{MeV}$ & $2 \mathrm{~cm}$ & 0.945 \\
\hline FLASH & $9 \mathrm{MeV}$ & $4 \mathrm{~cm}$ & 0.921 \\
\hline
\end{tabular}

Table 2. Values of the coefficients $\alpha$ and $\beta$ in the equation $Y=a D+\beta D^{2}$ and $\alpha / \beta$ quotients which gives the dose-response curves for each dose rate.

\begin{tabular}{llll} 
Dose rate & $\boldsymbol{\alpha} \pm$ SEx10 & $\boldsymbol{\beta} \pm$ SEx10 & $\boldsymbol{\alpha} / \boldsymbol{\beta}^{*} \pm$ SE \\
\hline $1 \mathrm{~Gy} / \mathrm{min}$ & $0.47 \pm 0.63$ & $1.20 \pm 0.10$ & $0.4 \pm 0.5$ \\
\hline $5 \mathrm{~Gy} / \mathrm{sec}$ & $1.35 \pm 0.52$ & $0.53 \pm 0.08$ & $2.6 \pm 1.1$ \\
\hline $50 \mathrm{~Gy} / \mathrm{sec}$ & $1.28 \pm 0.57$ & $0.72 \pm 0.09$ & $1.8 \pm 0.8$ \\
\hline $600 \mathrm{~Gy} / \mathrm{sec}$ & $1.57 \pm 0.44$ & $0.18 \pm 0.07$ & $8.7 \pm 4.2$
\end{tabular}

* $\alpha / \beta$ quotient is the dose at which contributions from single-track (a coefficient) and double-track ( $\beta$ coefficient) events are equal

\section{Figures}




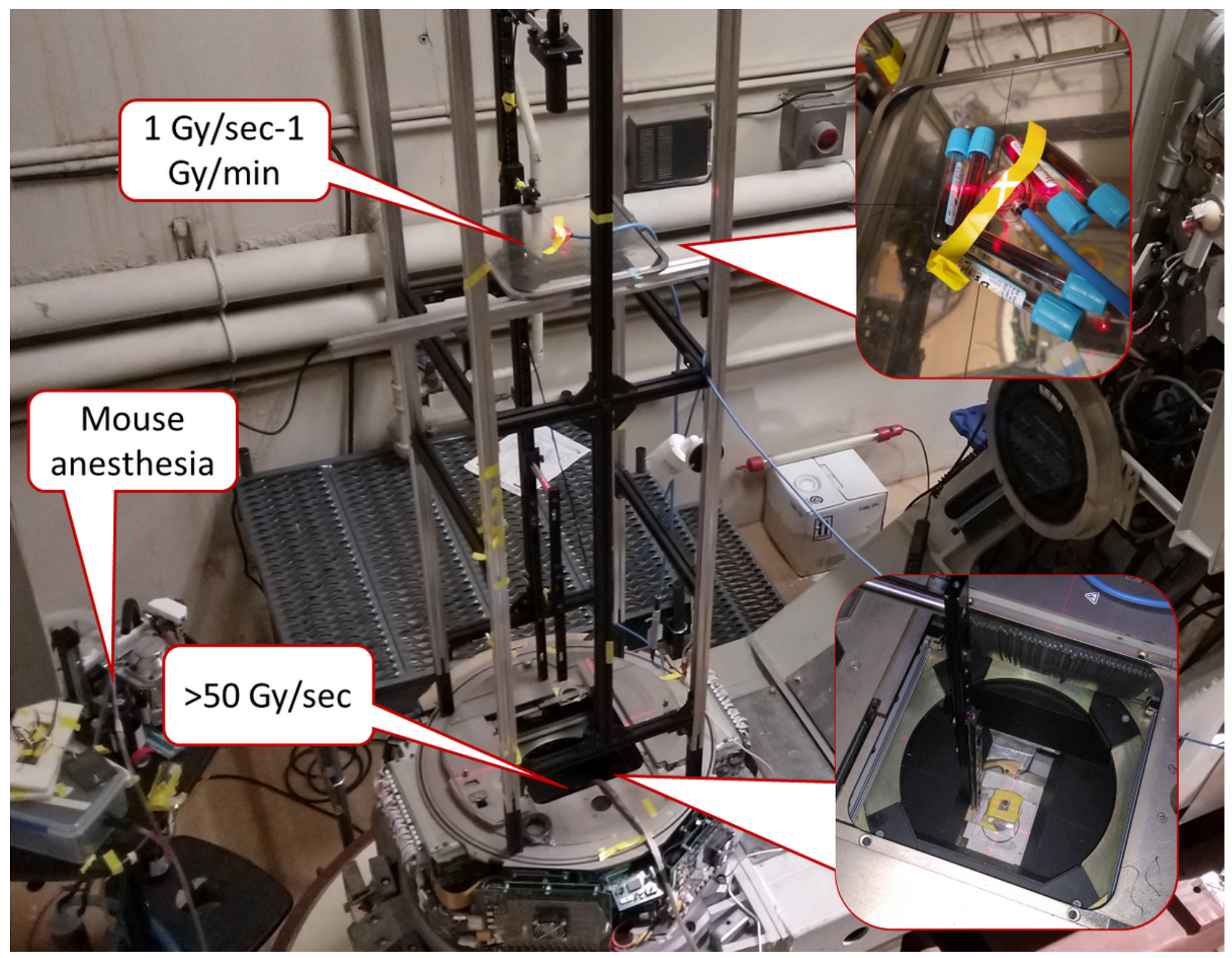

Figure 1

photo of the FLASH platform

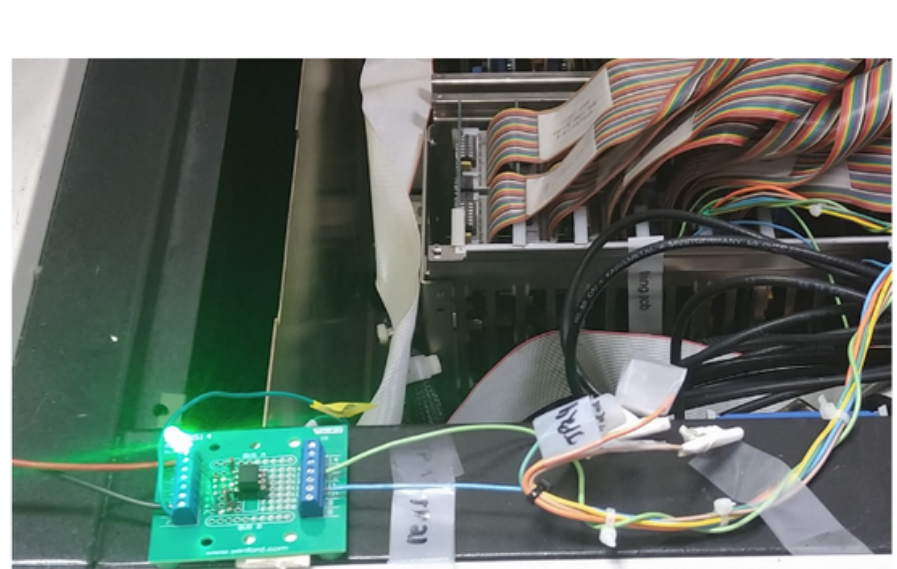

a)

b)

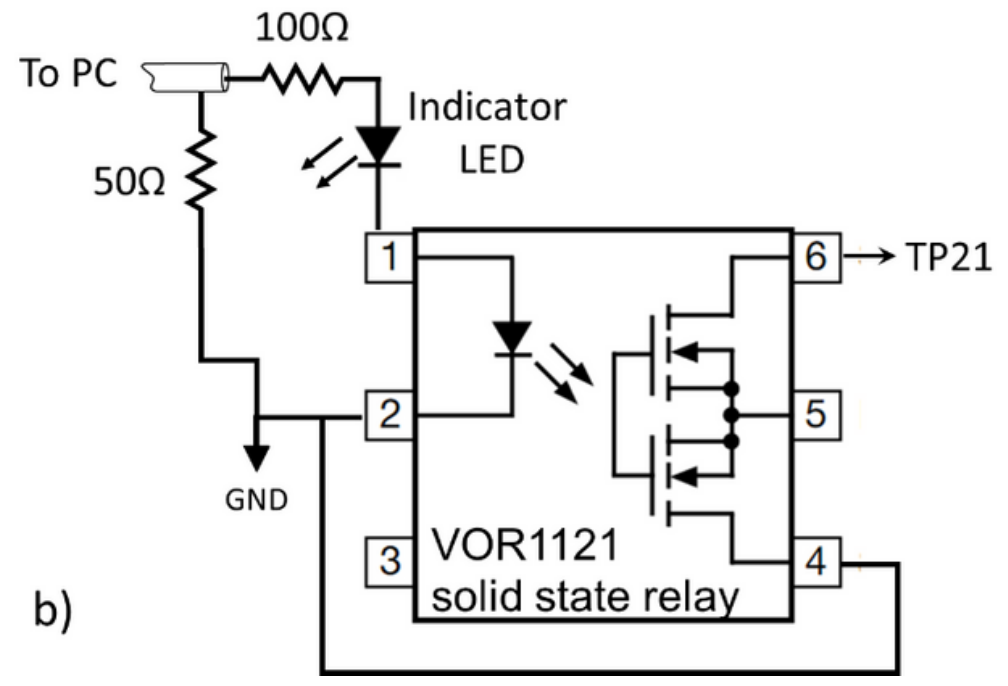


Figure 2

a) photo and b) schematic of the interface circuit between the PC and the Clinac timing card. 5V from the $\mathrm{PC}$ enables beam.

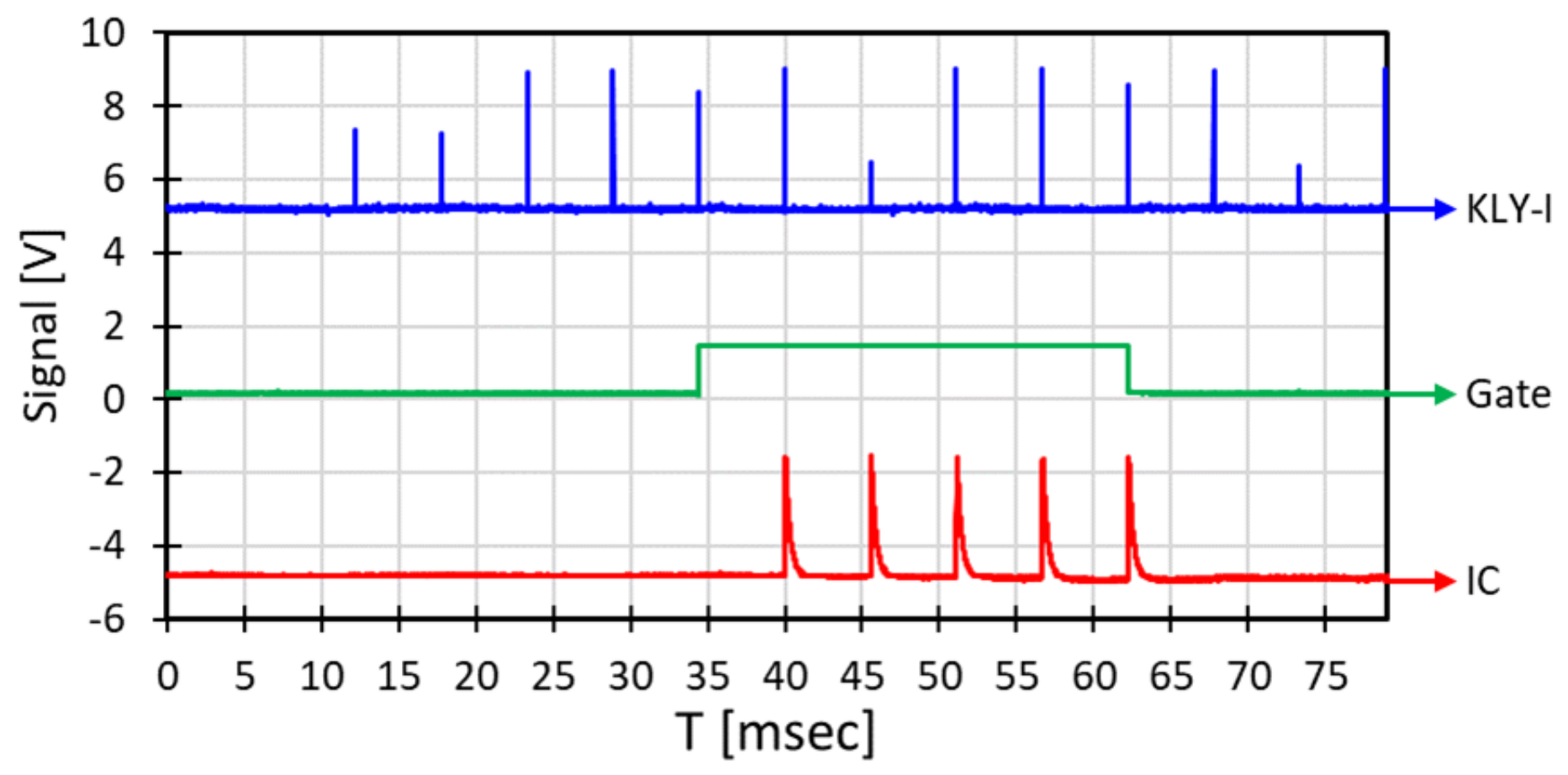

Figure 3

Oscilloscope trace of KLY-I, the CTR08-generated beam enable signal (Gate) and the built in ion chamber (IC). This irradiation was programmed to deliver 5 pulses after a delay of 5 pulses $(25 \mathrm{msec})$. Variation in KLY-I pulse height is due to undersampling - pulses are all 5V. KLY-I and IC traces offset by $\pm 5 \mathrm{~V}$ to facilitate viewing.

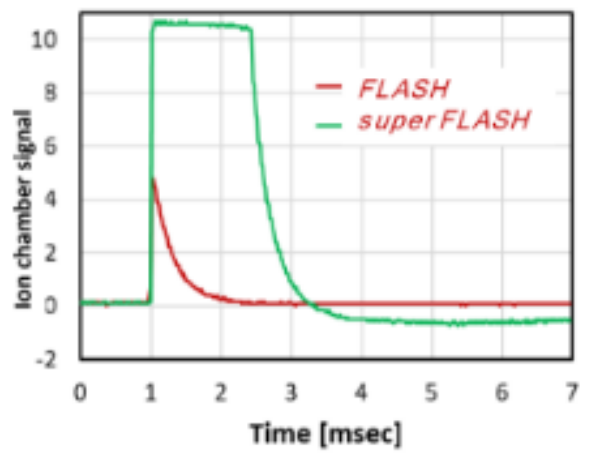

Figure 4: signal from the built in ion chamber for the two operation modes.

Figure 4 
signal from the built in ion chamber for the two operation modes.

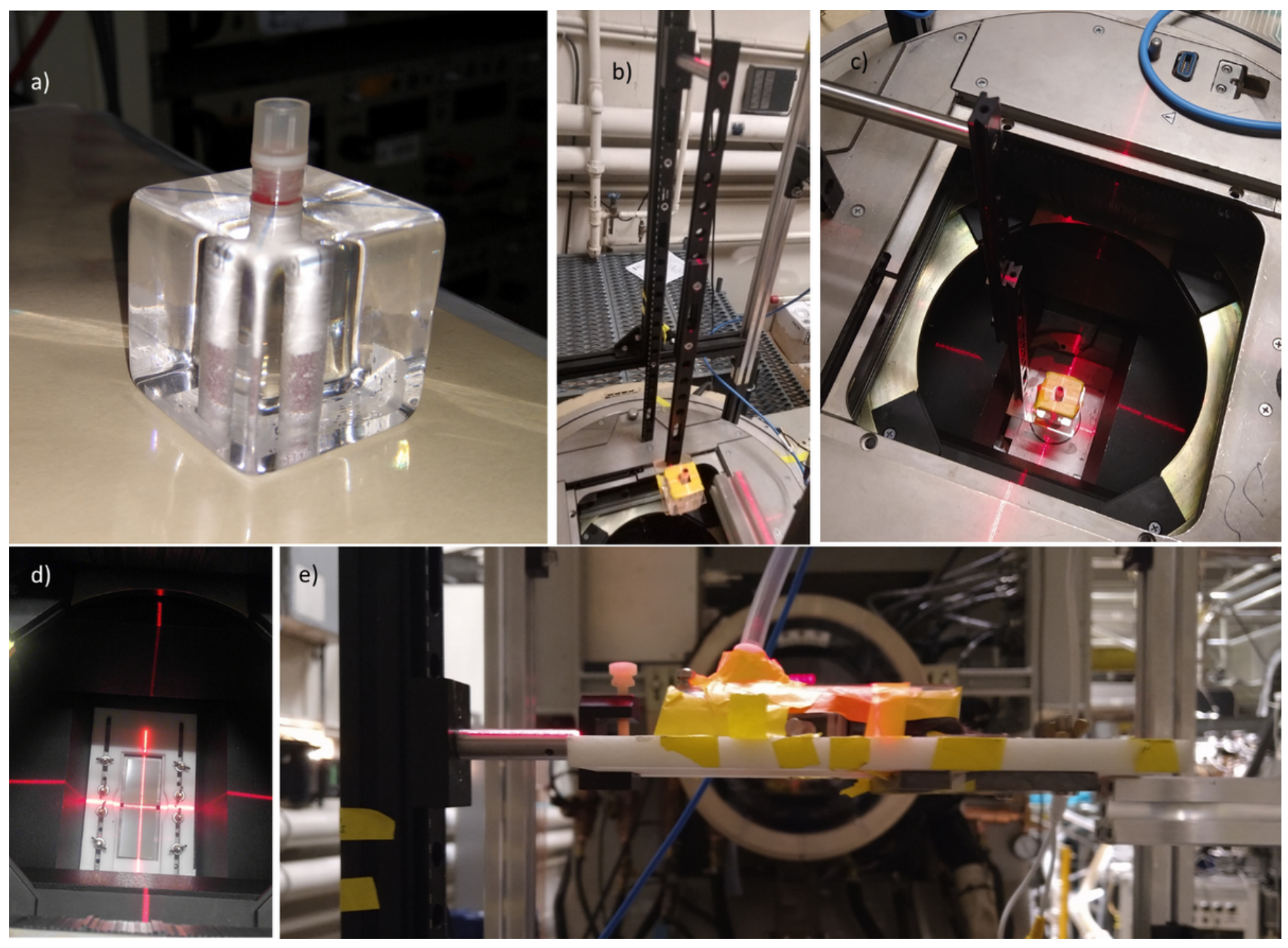

Figure 5

a) holder for matrix tube at b) $5 \mathrm{~Gy} / \mathrm{sec}$ position and c) $100 \mathrm{~Gy} / \mathrm{sec}$ position. d) shielded holder for mice.

e) shielded holder with anesthetized mouse being irradiated hemi-body at $10 \mathrm{~Gy} / \mathrm{sec}$ position. 


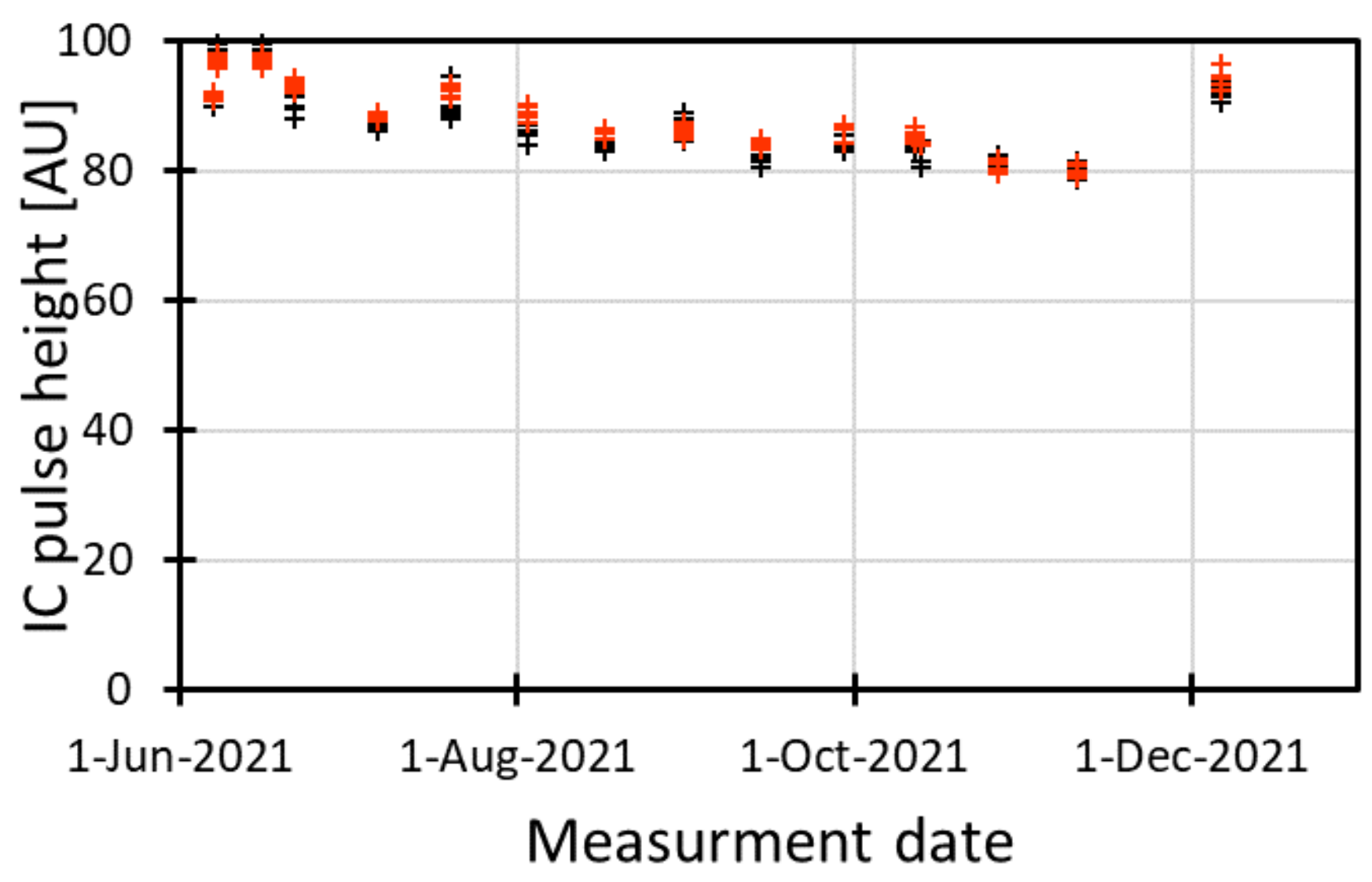

Figure 6

Average pulse height for 2 pulse irradiations (red) and 3 pulse irradiations (black) over 6 months of experiments. 


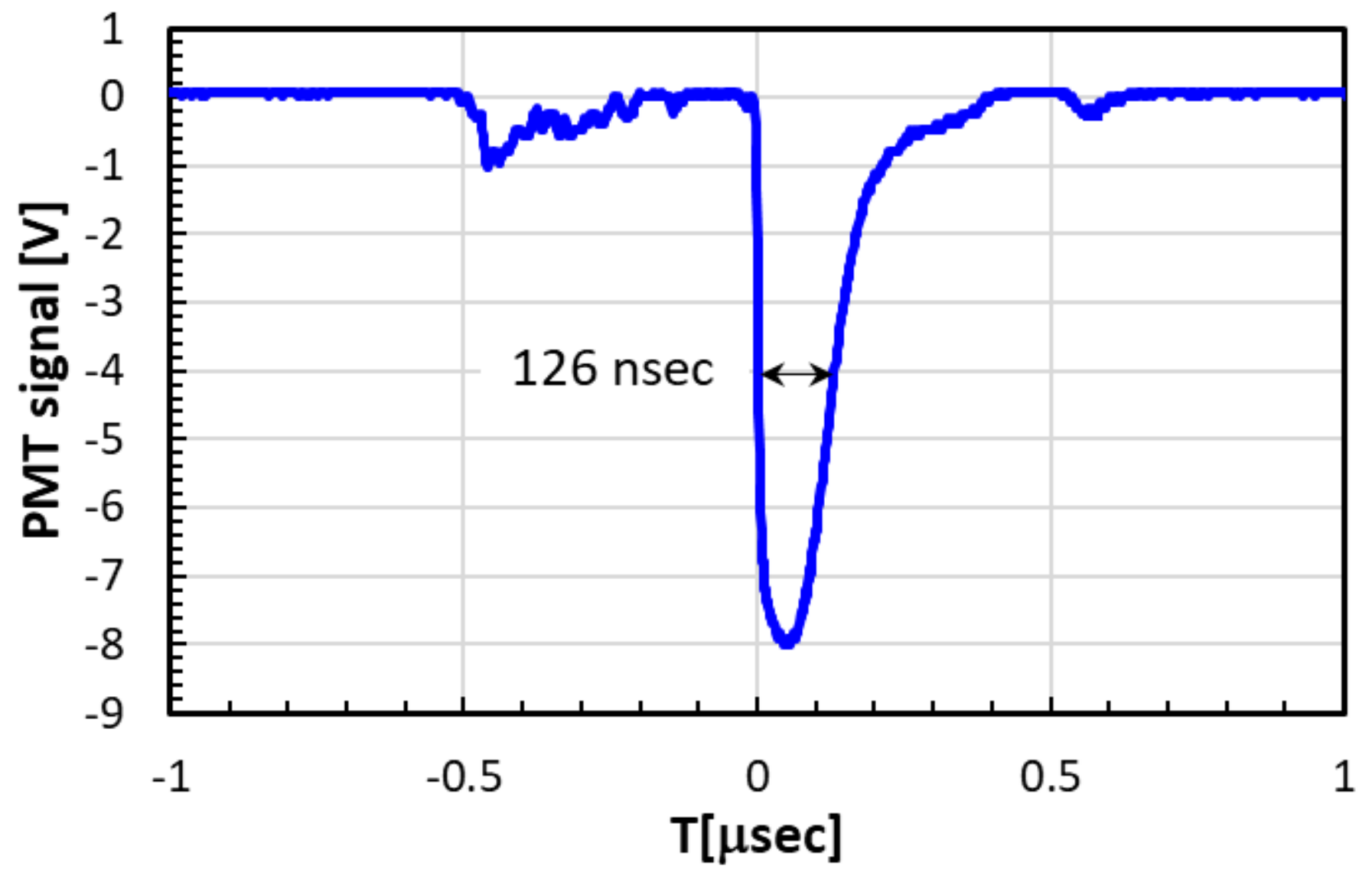

Figure 7

Oscilloscope trace of a single Clinac pulse measured directly using a scintillator and PMT tube.

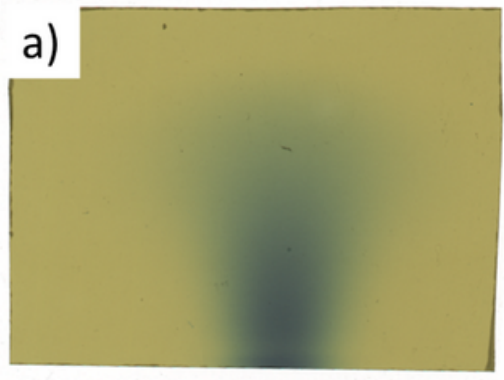

b)

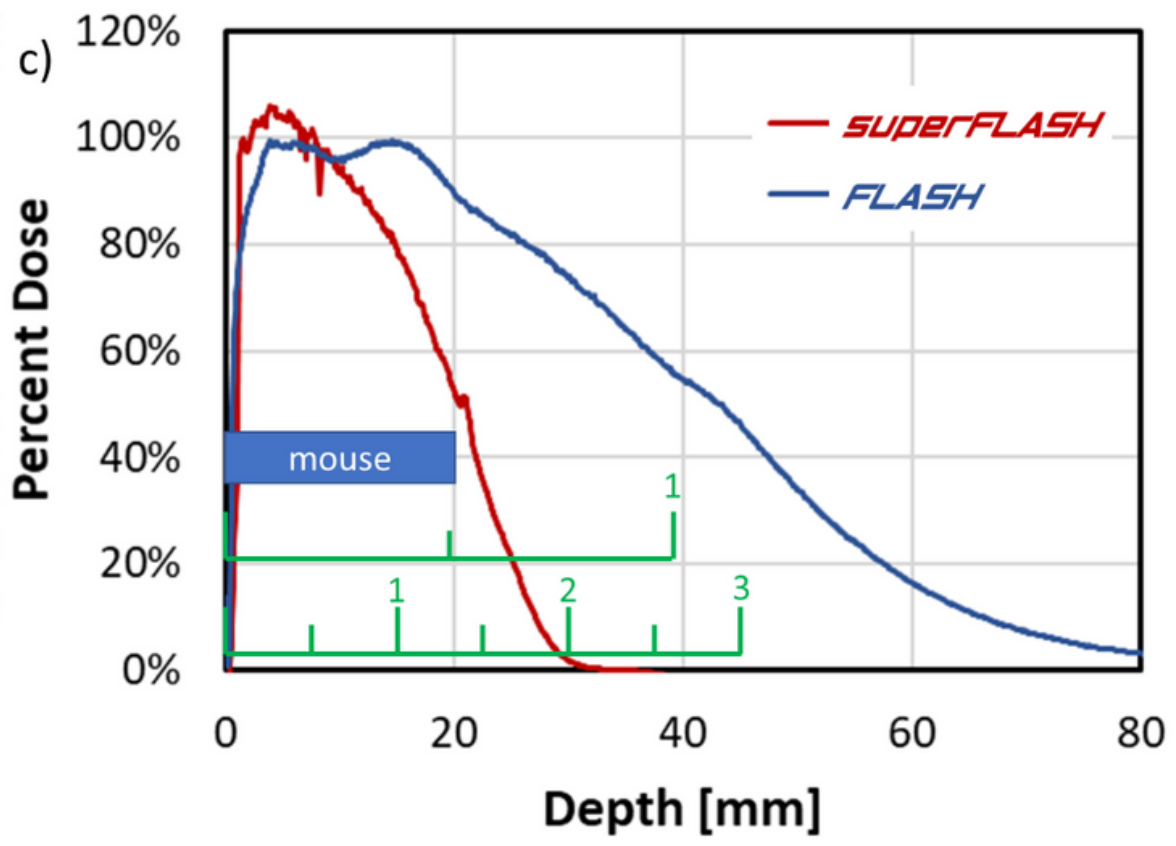

Figure 8 
Percent Depth Dose measurement. a)EBT3 and b) OC1 film irradiated parallel to 9 and $6 \mathrm{MeV}$ beams, respectively. Scale bar is $4 \times 1 \mathrm{~cm}$. c) Dose measured along beam. The scale bars show the thickness of a mouse, of $1 \mathrm{ml}$ liquid in a matrix tube $(6.3 \mathrm{~mm} \mathrm{ID})$ and $3 \mathrm{ml}$ liquid in a vacutainer (11 $\mathrm{mm} \mathrm{ID})$.

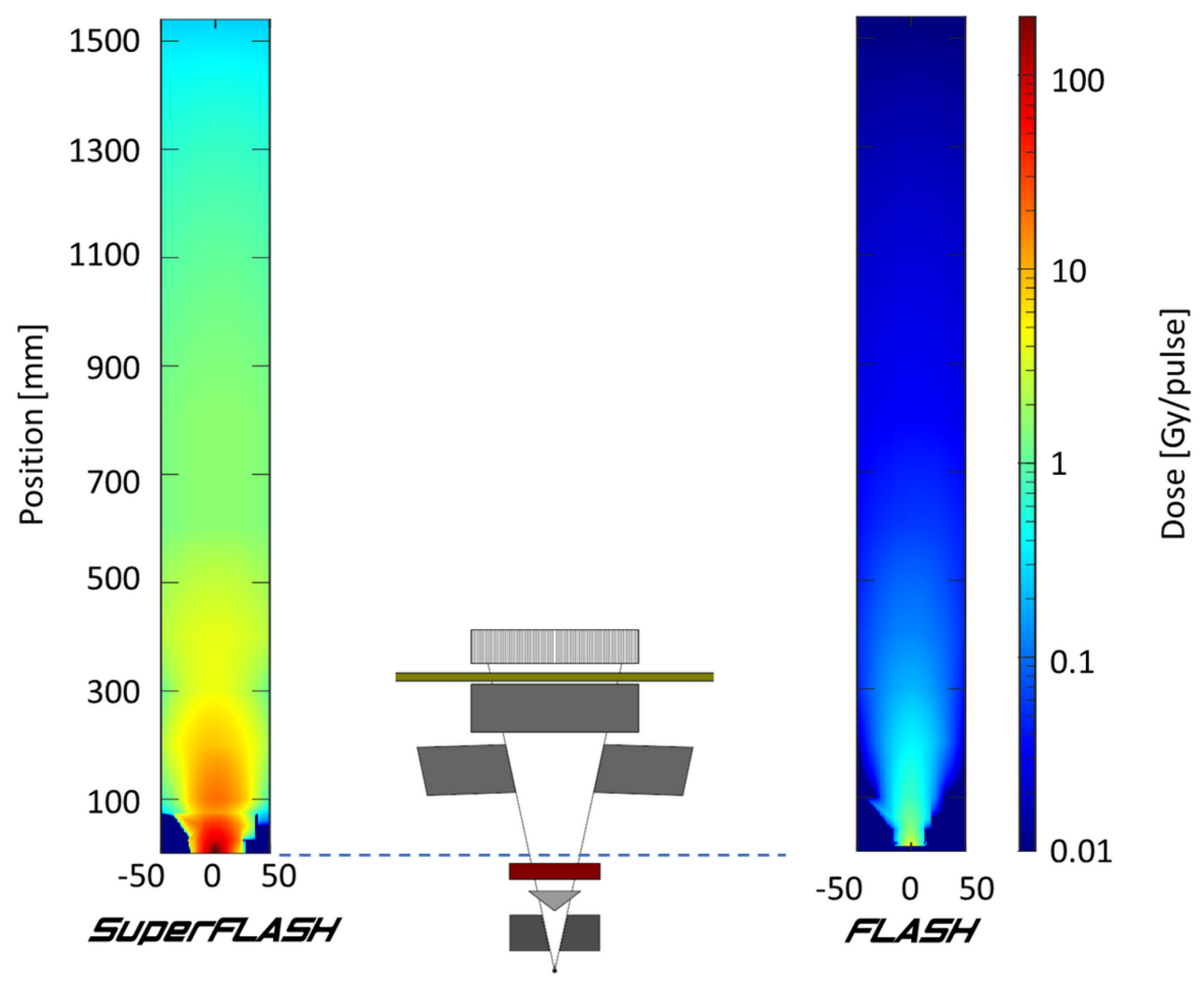

Figure 9

heat map of dose rate in the two operation modes. 


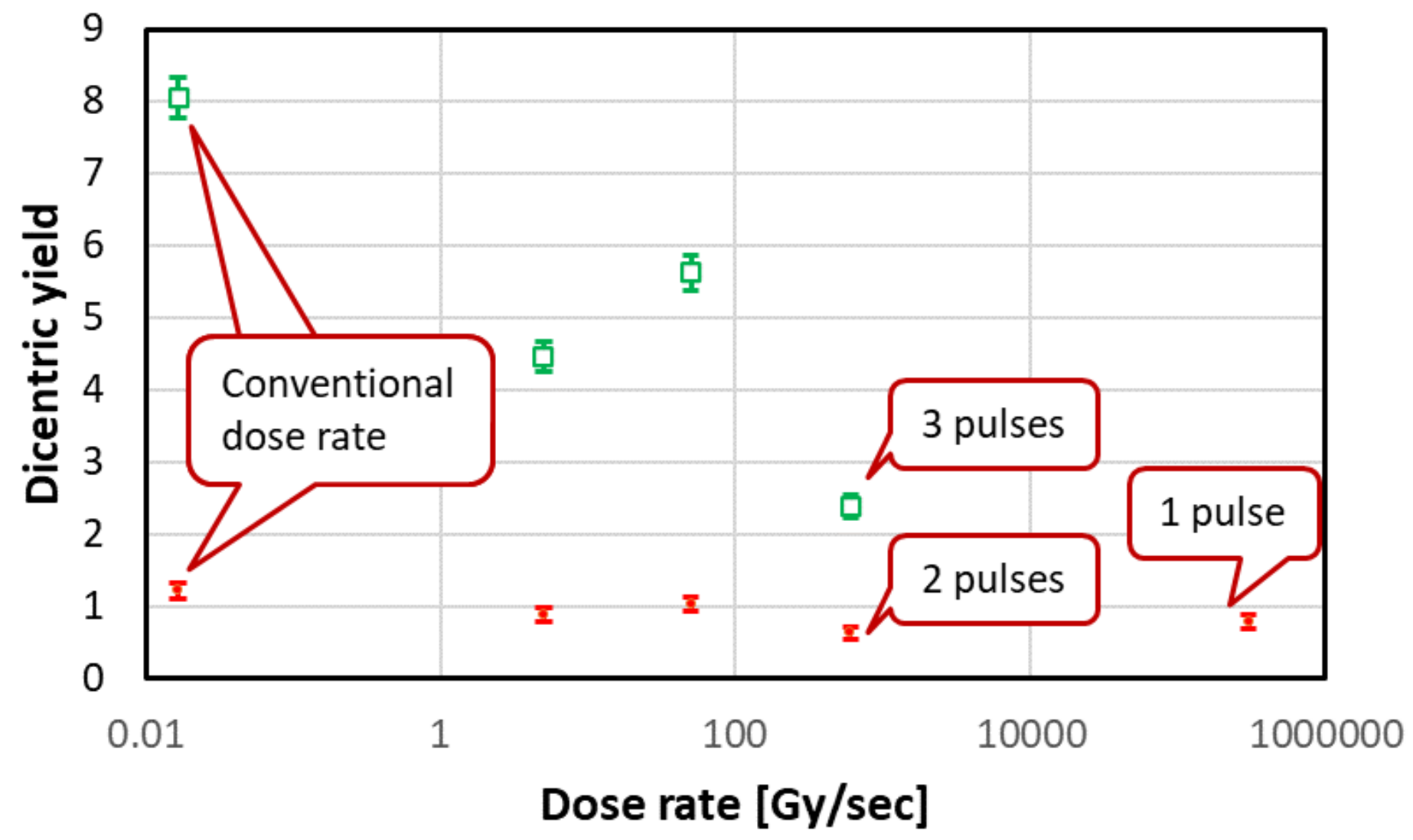

Figure 10

dicentric yields for $3 \mathrm{~Gy}$ (red closed symbols) and $8 \mathrm{~Gy}$ (green open symbols) irradiations at different dose rates. No dicentrics or multicentrics (yield $=0 \pm 0$ ) seen in control samples.
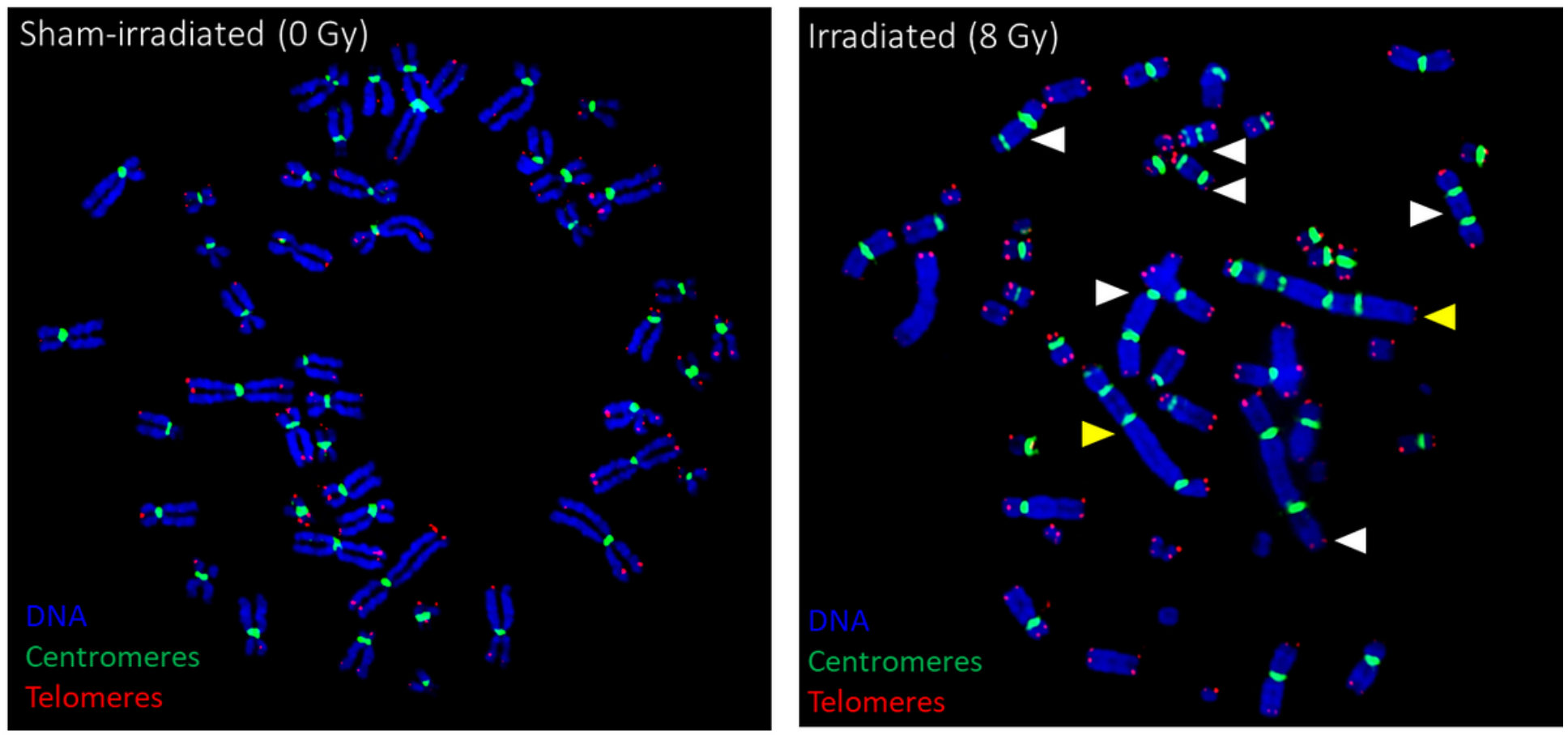
Figure 11

PNA-FISH-based detection of dicentric chromosomes: representative images of control (left) or irradiated to $8 \mathrm{~Gy}$ (right) lymphocytes. Aberant chromosomes are indicated by white (dicentrics, centromere number $=2$ ) or yellow (multicentrics, centromere numbers $>2$ ) arrows.

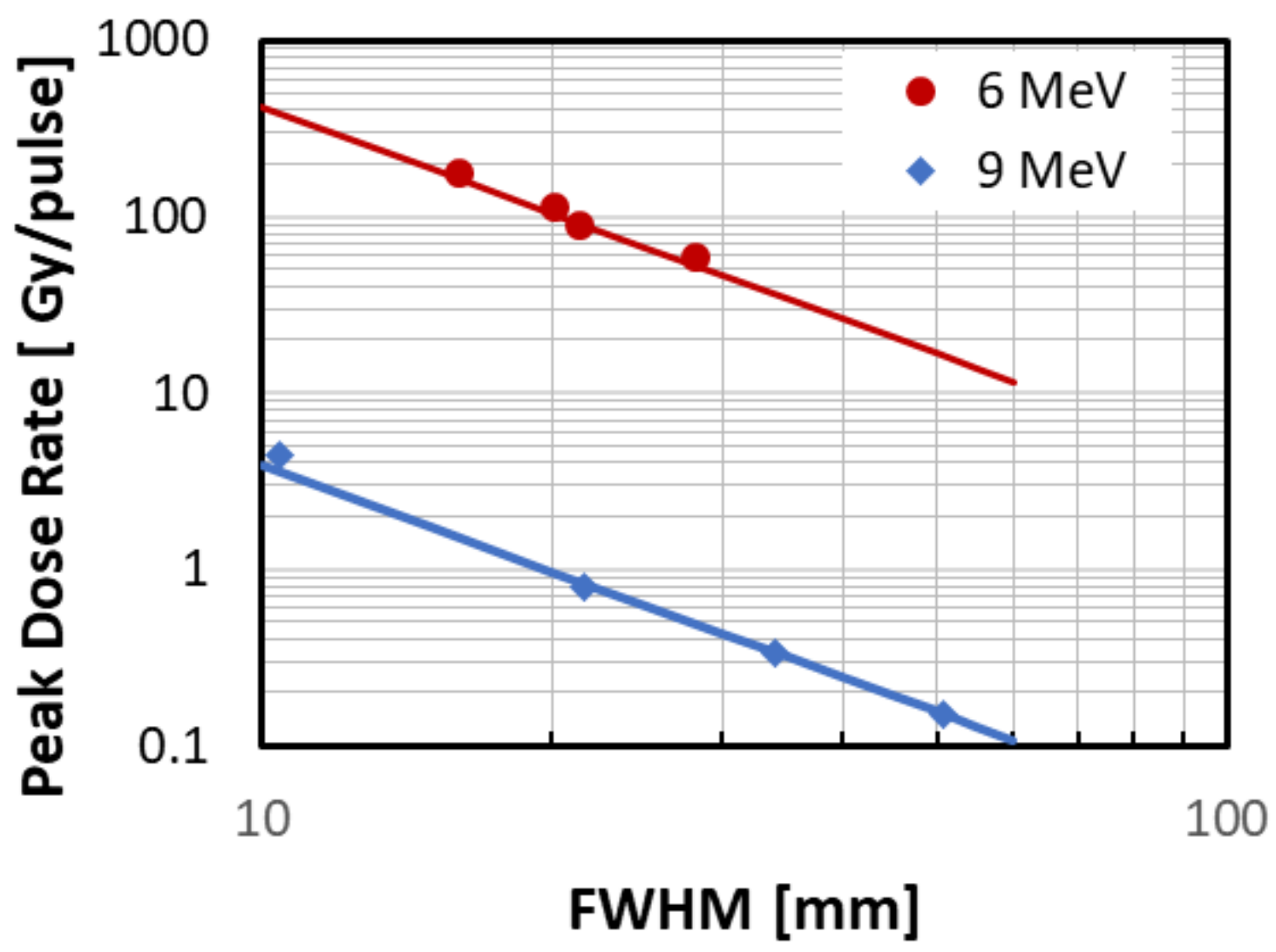

Figure 12

peak dose rate vs Full Width at Half Max (FWHM) in FLASH and superFLASH modes

\section{Supplementary Files}

This is a list of supplementary files associated with this preprint. Click to download.

- SupplementaryData.docx 